\title{
Enem inclusivo: identificando técnicas para a realização assistida pelo computador
}

\author{
Hedi C. Minin ${ }^{1}$, Daniela G. Trevisan ${ }^{1}$, José Viterbo ${ }^{1}$ \\ ${ }^{1}$ Instituto de Computação - Universidade Federal Fluminense (UFF) \\ Niterói - RJ - Brazil \\ $\{$ hminin, daniela, viterbo\}@ic.uff.br
}

\begin{abstract}
This work investigates techniques to the development of a delivery tool of Enem test through the computer in order to meet the specific accessibility needs of candidates with full and partial visual impairment. After the identification of appropriate techniques, we conducted an experimental analysis to determine if they are able to properly deliver the content of the exam to this audience. Finally, we propose the application of the techniques identified creating a functional prototype that will serve to accomplish testing with real users, necessary for the identification of possible accessibility and usability problems.
\end{abstract}

Resumo. Este trabalho investiga técnicas apropriadas para o desenvolvimento de uma ferramenta de entrega da prova do Enem por meio do computador, de forma a atender as necessidades de acessibilidade específicas de candidatos com deficiência visual total e parcial. Após a identificação das técnicas apropriadas, realizamos uma análise experimental para determinar se estas são capazes de entregar corretamente o conteúdo do exame a este público. Por fim, propomos a aplicação das técnicas identificadas na criação de um protótipo funcional que servirá para realizarmos testes com usuários reais, necessários para a identificação de possíveis problemas não somente de acessibilidade, mas também de usabilidade.

\section{Introdução}

O Exame Nacional do Ensino Médio (Enem) foi criado pelo Instituto Nacional de Estudos e Pesquisas Educacionais (INEP) em 1998 para ser aplicado anualmente aos alunos concluintes da escolaridade básica e aos egressos. Seu objetivo fundamental é o de avaliar o desempenho do estudante ao término deste nível de ensino, medindo o desenvolvimento de competências e habilidades necessárias ao exercício pleno da cidadania [Ministério da Educação 2002]. Atualmente o resultado do Enem também é utilizado como forma de seleção unificada nos processos seletivos das Instituições Federais de Ensino Superior (IFES) e como critério de seleção de alunos que serão beneficiados com programas governamentais, como o Programa Universidade para Todos (ProUni).

Ciente da importância da utilização do resultado do Enem não apenas como um indicador, mas como parâmetro de seleção de alunos ingressantes em Instituições Federais de Ensino em todo o Brasil e de beneficiários de programas governamentais, o Ministério da Educação (MEC) busca, a cada edição do exame, aperfeiçoar o 
atendimento a candidatos com algum tipo de deficiência, por meio da oferta de profissionais especializados e de recursos de acessibilidade. Para atender deficientes visuais o MEC oferece provas adaptadas (em braile e ampliadas) e auxílio ledor, onde uma pessoa realiza a leitura da prova para o candidato [Ministério da Educação 2012]. Dados disponibilizados pelo INEP mostram que a procura por estes recursos e serviços têm aumentado a cada edição do exame. Entre 2011 e 2014, por exemplo, houve um aumento de $235 \%$ no número de solicitações de auxílio ledor e de $132 \%$ no número de solicitações de prova ampliada [INEP 2014].

Apesar dos esforços aplicados na promoção de acessibilidade, alguns candidatos com necessidades especiais, como deficientes visuais, relatam experiências frustrantes durante a realização do exame. A falta de preparação dos ledores e o cansaço são algumas dificuldades relatadas [Leonardo 2014]. Neste cenário, a utilização do computador como meio de entrega de testes para pessoas com necessidades especiais é citada como uma alternativa capaz de diminuir barreias e de promover oportunidades, permitindo-lhes a demonstração de seu real potencial [Ketterlin-Geller 2005] [Scheuermann e Pereira 2008][Thurlow et al 2010].

Para Thurlow et al, testes baseados em computadores (CBT-Computer-based Test) apresentam vantagens significativas e podem ser mais acessíveis se comparados a testes realizados utilizando lápis e papel. A possibilidade de ajustar as propriedades de exibição e de realizar o teste sem o auxílio de outra pessoa, por exemplo, são algumas vantagens citadas. Entretanto, apesar de proporcionar benefícios expressivos, o uso do computador como meio de entrega de testes pode se apresentar como uma experiência frustrante e influenciar negativamente na demonstração de conhecimentos, competências e habilidades de candidatos com algum tipo de deficiência, caso a ferramenta de entrega não seja cuidadosamente projetada [Thurlow et al 2010].

Visando a inclusão de deficientes visuais junto ao Enem e diante das limitações encontradas nas soluções existentes, este trabalho procura identificar técnicas apropriadas para o desenvolvimento de uma ferramenta de entrega da prova do Enem por meio do computador. Apesar de encontrarmos candidatos com diversos tipos de deficiência, neste primeiro momento concentramos nossos esforços na busca por soluções que atendam as necessidades de acessibilidade específicas de candidatos com deficiência visual total ou parcial, uma vez que a maioria das solicitações de atendimento especializado no Enem parte deste público.

\section{Trabalhos relacionados}

Dentre os trabalhos sobre acessibilidade em CBTs que incluem pessoas com deficiência visual, os relatórios de pesquisa da ETS (Educational Testing Service), apresentam estudos relevantes sobre o tema. A ETS é uma organização sem fins lucrativos que desenvolve e administra mais de 50 milhões de exames por ano, dentre os quais o mais conhecido é TOEFL (Test of English as a Foreign Language).

Um destes trabalhos procura identificar pontos fortes e fracos dos sistemas de entrega do TOEFL no cumprimento dos requisitos de acesso por pessoas com os seis diferentes tipos de deficiência, incluindo cegueira e baixa visão. Neste trabalho os autores exploram três abordagens de entrega de texto, descrevendo o seu desenvolvimento e avaliações qualitativas. Tais abordagens são: o Self-Voicing Test 
(SVT), que provê um mecanismo interno de conversão de texto em voz (TTS - text-tospeech), dispensando a utilização de softwares externos, como os softwares leitores de tela; o HTML-Form System (HFS), desenvolvido em HTML (HyperText Markup Language) e que foi concebido para ser compatível com softwares leitores de tela; e o Visually Oriented System (VOS), orientado visualmente e destinado a pessoas surdas ou que não possuam deficiência [Hansen, Forer e Lee 2004].

Após avaliar as duas primeiras abordagens, a maioria dos participantes cegos ou com baixa visão recomendaram fortemente o SVT e 70\% participantes que já haviam utilizado ledores em testes anteriores disseram preferir utilizar o computador a ledores. Uma vantagem citada pelos participantes é a possibilidade de realizar o teste de forma independente.

Para incluir alunos com deficiência visual no GRE revised General Test, um teste para admissão de alunos na pós-graduação, a ETS desenvolveu uma ferramenta de entrega de testes por meio do computador que provê um mecanismo de sintetização de voz (self-voicing), dispensando o uso de outros softwares leitores de tela [Frankel e Kirsh 2014]. Os usuários navegam pelo teste utilizando somente o teclado e teclas de atalho, que foram projetadas para ser o mais parecido possível com os atalhos utilizados por leitores de tela, como o JAWS (Job Access With Speech). Para os usuários com baixa visão, a ferramenta permite que propriedades visuais, como o tamanho e cor do texto, sejam alteradas. A avaliação desta ferramenta com usuários reais revelou dois problemas significativos: (1) Algumas palavras não eram pronunciadas de forma suficientemente clara; e (2) Alguns usuários relataram dificuldades para utilizar atalhos de que eram diferentes daqueles utilizados pelos leitores de tela, e que já estavam acostumados a utilizar.

Thurlow et al [2010] apresentam questões a serem consideradas na concepção e implementação de testes baseados em computadores para incluir pessoas com deficiência. Os autores mencionam que, em testes baseados em papel e lápis deficientes visuais podem contar, por exemplo, com o auxílio de ledores, mas que a qualidade destes ledores varia consideravelmente de um indivíduo para outro: ledores podem pronunciar palavras de forma errada, utilizar entonação inadequada e até influenciar nas respostas dos alunos. Neste cenário, eles afirmam que os CBTs podem oferecer consistência utilizando voz digitalizada ou conversão de texto para voz para entregar o conteúdo do teste.

Apesar das vantagens, os autores enfatizam que, para alunos com deficiências visuais graves ou cegueira, os testes baseados em computadores podem ser menos acessíveis que testes realizados com papel e lápis. Eles descrevem alguns desafios na utilização de testes baseados em computadores por deficientes visuais: (1) alguns testes baseados em computadores podem exigir um o esforço cognitivo maior se comparado a testes semelhantes realizados com papel e lápis; (2) alguns alunos podem nunca ter aprendido a utilizar computadores ou não ter habilidades motoras para utilizarem um teclado e/ou mouse com sucesso; e (3) a tecnologia assistiva ou o dispositivo de entrega de conteúdo que o aluno está acostumado a usar pode não estar disponível na ferramenta de entrega do teste. 


\section{Metodologia}

Como o público alvo possui necessidades específicas, que variam conforme o tipo de deficiência, o trabalho de identificação das técnicas apropriadas para entrega do conteúdo do exame foi realizado em três etapas: (1) identificação de técnicas para atender pessoas com deficiência visual total; (2) identificação de técnicas para atender pessoas com deficiência visual parcial; (3) análise experimental de como as técnicas identificadas lidam com os diferentes tipos de conteúdos do exame (textos, tabelas, gráficos, equações, imagens), necessária para detectar possíveis inconsistências e problemas de acessibilidade. Como resultado destas etapas propomos a aplicação das técnicas identificadas no desenvolvimento de um protótipo funcional que será alvo de futura avaliação com usuários.

O levantamento de informações necessárias para conduzir a identificação destas técnicas foi realizado por meio de pesquisa bibliográfica e entrevista semi-estruturada com três deficientes visuais, dois destes profissionais que atuam no Instituto Benjamin Constant (IBC). Para Nielsen [1993], as entrevistas são métodos úteis para o estudo de como os usuários utilizam os sistemas e quais as características que eles particularmente apreciam mais ou menos, bem como identificar os principais problemas enfrentados por estes usuários.

\section{Seleção de técnicas}

A seleção das técnicas apropriadas para entrega do exame foi realizada considerando as características do exame atual, realizado com lápis e papel, e as necessidades de acessibilidade de pessoas com deficiência visual total ou parcial, que foram identificadas tanto na literatura quanto nas entrevistas realizadas.

\subsection{Técnicas para conversão do texto em áudio}

Para entregar o conteúdo do exame a pessoas que possuem deficiência visual total - ou mesmo parcial severa - é necessário que o conteúdo do exame seja convertido em áudio. Este conteúdo pode ser dividido em duas categorias: conteúdo estático, que não sofre alterações ao longo do exame, como o conteúdo das instruções e questões, e conteúdo dinâmico, que sofre alterações ao longo do exame e é criado pelo candidato, como o conteúdo do rascunho e redação.

De acordo com a ETS [2010], a entrega do exame em formato de áudio pode ser realizada por meio de áudio pré-gravado, normalmente gravado em CDs (Compact Disc), ledores ou ainda texto convertido em fala por um sintetizador de voz. Em uma ferramenta computacional, somente duas destas abordagens podem ser adotadas: o áudio pré-gravado e o sintetizador de voz. Considerando que a sintetização de voz pode ser realizada tanto por um software externo quanto pela própria ferramenta de entrega do exame, discutimos o uso de três abordagens: (1) áudio pré-gravado; (2) sintetização de voz pela própria ferramenta (self-voicing); e (3) sintetização de voz por meio de um software externo, neste caso, leitores de tela.

Durante as entrevistas, constatamos que a conversão do conteúdo do exame em fala por si só não atenderia plenamente as necessidades deste público, uma vez que o deficiente visual não apenas atua passivamente neste processo como ouvinte, mas também necessita interagir com o conteúdo, decidindo como e o que ouvir. Desta forma, 
as necessidades apontadas pelos entrevistados, além de características do exame, sugeriram sete requisitos que a abordagem utilizada para conversão do conteúdo do exame em fala deve atender. Tais requisitos e seu suporte por cada uma das três abordagens são apresentados na Tabela 1 .

Inicialmente consideramos o uso da primeira abordagem, em que uma aplicação self-voicing entregaria o conteúdo estático por meio de arquivos de áudio pré-gravado. Nesta abordagem, a conversão do texto em fala é realizada previamente, o que torna possível a identificação e correção de possíveis inconsistências. Entretanto, tal abordagem apresentou, dentre outras, duas limitações significativas: a primeira é limitar-se ao conteúdo estático, o que implica na utilização de um mecanismo sintetizador de voz para o conteúdo dinâmico, e a segunda é não ser flexível o suficiente para permitir a navegação pelo texto, como observou uma participante: "Se eu não entender uma palavra e precisar soletrá-la, como farei?", que complementou: "Da mesma forma que vocês - videntes - varrem um parágrafo com os olhos para encontrar uma palavra, nós precisamos fazê-lo, navegando pelo texto.”.

Devido a estas limitações, o uso da segunda abordagem, uma aplicação selfvoicing que faz uso de um conversor de texto em fala, foi então considerada. Esta abordagem é a mesma utilizada por Frankel e Kirsh [2014]. Para evitar que usuários acostumados a utilizar um software leitor de tela vivenciem as dificuldades citadas pelos autores, a ferramenta de entrega do exame deveria implementar diversos recursos presentes em um software leitor de tela e que contemplam a maioria dos requisitos apresentados na Tabela 1. Entretanto, para Hersh e Johnson [2008], em muitos aspectos, a experiência do usuário com uma aplicação self-voicing é muito semelhante ao da utilização de um leitor de tela com um aplicativo padrão. Dolan et al [2010] afirmam que os leitores de tela têm a vantagem de serem mais customizáveis e muitos usuários cegos preferem usar aplicativos comuns ao invés de aplicações fora do padrão.

Neste aspecto, todos os entrevistados concordaram que utilizar a terceira abordagem - software leitor de telas - é uma solução mais apropriada, ao invés de recriar as mesmas funcionalidades na ferramenta de entrega do exame, uma vez que deficientes visuais usuários de computadores muito provavelmente já fazem uso deste tipo de software.

Tabela 1. Comparação do suporte aos requisitos para conversão do conteúdo do exame em fala ( $\mathrm{S}=$ atende, $\mathrm{N}=$ não atende)

\begin{tabular}{|l|c|c|c|}
\hline \multirow{2}{*}{\multicolumn{1}{|c|}{ Requisito }} & \multicolumn{2}{|c|}{ Self-voicing } & Leitor de telas \\
\cline { 2 - 4 } & $\begin{array}{c}\text { Áudio pré- } \\
\text { gravado }\end{array}$ & Sintetizador & Sintetizador \\
\hline Alterar voz em tempo de execução & $\mathrm{N}$ & $\mathrm{S}^{1}$ & $\mathrm{~S}$ \\
\hline $\begin{array}{l}\text { Alterar idioma em tempo de } \\
\text { execução }\end{array}$ & $\mathrm{N}$ & $\mathrm{S}^{2}$ & $\mathrm{~S}^{2}$ \\
\hline $\begin{array}{l}\text { Alterar propriedades da voz (Ex. } \\
\text { tom) }\end{array}$ & $\mathrm{S}^{1}$ & $\mathrm{~S}^{1}$ & $\mathrm{~S}$ \\
\hline Controle total sobre a fala & $\mathrm{S}$ & $\mathrm{S}$ & $\mathrm{S}^{3}$ \\
\hline
\end{tabular}




\begin{tabular}{|l|c|c|c|}
\hline (o que e como deve ser falado) & & & \\
\hline $\begin{array}{l}\text { Flexibilidade (Ex. navegar por } \\
\text { sentenças, soletrar palavras) }\end{array}$ & $\mathrm{N}$ & $\mathrm{S}^{1}$ & $\mathrm{~S}$ \\
\hline Garantia de pronúncia correta & $\mathrm{S}$ & $\mathrm{N}^{4}$ & $\mathrm{~N}^{4}$ \\
\hline Falar a entrada do usuário & $\mathrm{N}$ & $\mathrm{S}^{1}$ & $\mathrm{~S}$ \\
\hline
\end{tabular}

1. Necessário que a ferramenta de entrega do exame implemente tais funcionalidades.

2. Necessário que o motor de sintetização suporte esta funcionalidade.

3. O leitor de telas pode dizer mais (ou menos) informações que o necessário [Hansen, Forer e Lee 2004].

4. Algumas palavras podem não ser pronunciadas corretamente [Frankel e Kirsh 2014].

\subsection{Técnicas para ampliação e personalização das propriedades de exibição}

Pessoas com deficiência visual que ainda possuem alguma capacidade para enxergar fazem uso de um software ampliador de tela para ter acesso às informações exibidas na tela. A finalidade de tal software é ampliar uma parte da tela de um sistema de computador para que ele se torne visível à pessoa com deficiência visual [Hersh e Johnson 2008]. Além da ampliação, estes softwares permitem que os usuários invertam ou ajuste as cores de acordo com sua necessidade [Pernice e Nielsen 2001].

Usuários de ampliadores de tela ampliam consideravelmente o texto, criando a necessidade de efetuar a rolagem da tela horizontalmente e verticalmente diversas vezes para ter acesso a todo o conteúdo presente na tela. Consequentemente, quanto mais conteúdo é apresentado na tela, mais tempo estes usuários irão demorar a concluir a leitura e mais difícil será sua recordação [Pernice e Nielsen 2001]. Hersh e Johnson [2008] observam ainda que o texto ampliado pode parecer bastante quadriculado. Esta situação pode ser observada na Figura 1, que apresenta o trecho de uma questão ampliado 5 vezes utilizando o ampliador de telas presente no Windows 7 .

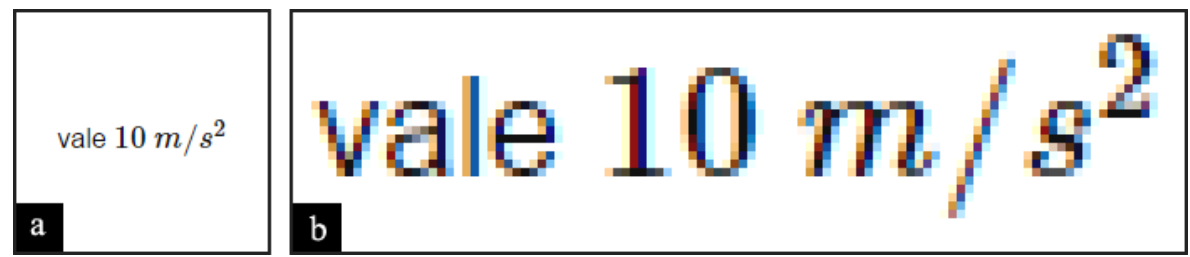

Figura 1. 0 texto original (a) se apresenta quadriculado (b) quando ampliado 5 vezes utilizando o ampliador de telas presente no Windows 7.

Devemos considerar ainda que as questões do exame não são compostas apenas por texto, mas também por gráficos, imagens, tabelas, equações e fórmulas. Desta forma, a ferramenta também deve oferecer meios para exibição e alteração de propriedades destes elementos. Dolan et al [2010] observam que, quando possível, os gráficos devem ser representados utilizando gráficos vetoriais escaláveis (SVG) ou equivalente, uma vez que este tipo de gráfico pode ser ampliado sem degradação.

Para evitar a necessidade de rolagem horizontal, estabelecemos que a tecnologia utilizada para desenvolvimento da ferramenta de entrega do exame deveria oferecer meios para que o usuário ajuste as propriedades de exibição sem a necessidade de utilizar um software externo. Neste sentido, identificamos três requisitos que tal tecnologia deve atender: (1) possibilitar a criação de um layout flexível o suficiente para 
que os elementos visuais se ajustem, quando possível, as diferentes resoluções de tela e aos diferentes níveis de ampliação; (2) permitir que os usuários ajustem as cores dos elementos visuais de acordo com sua preferência; e (3) suportar a exibição de gráficos vetoriais.

Inicialmente consideramos o desenvolvimento de uma aplicação desktop escrita em Java para a entrega do exame, porém, tal abordagem apresentou limitações quanto à flexibilidade necessária para exibição do conteúdo e personalização de algumas propriedades de exibição. Logo, devido às limitações encontradas, consideramos o uso de uma aplicação Web desenvolvida em HTML 5 e executada em um navegador Web. Esta abordagem se apresentou como a alternativa que melhor atendeu aos requisitos expostos: possibilita a criação de layout flexível utilizando CSS (Cascading Style Sheets), ampliação de elementos e do texto sem degradação, alteração de cores, suporte nativo para exibição de gráficos vetoriais e suporte nativo para MathML (Mathematical Markup Language) - uma linguagem para apresentação de símbolos e fórmulas matemáticas.

A Figura 2 ilustra parte de uma questão ampliada 4 vezes em uma aplicação Web utilizando CSS e a mesma questão ampliada na mesma proporção em uma aplicação desktop utilizando o ampliador de telas presente no Windows 7. Podemos notar que na aplicação Web o ajuste do texto dispensa a necessidade de rolagem horizontal.
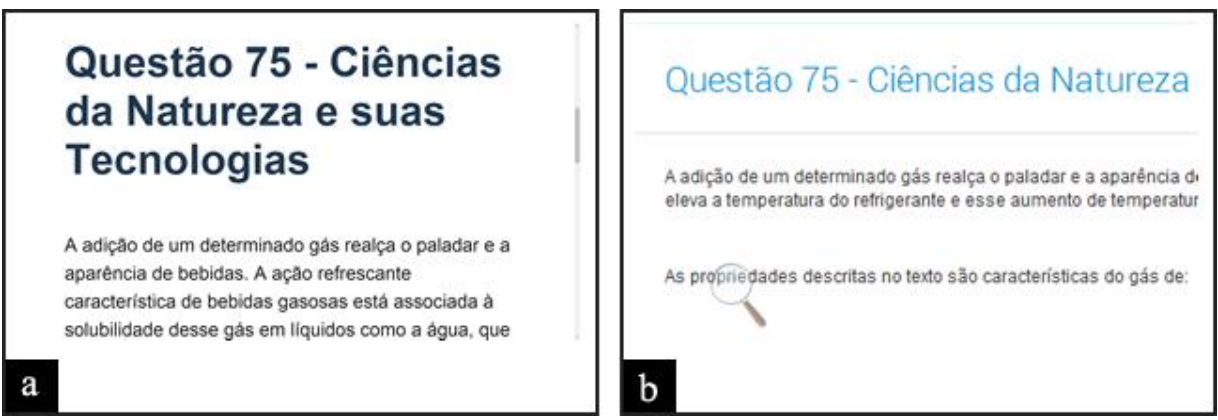

Figura 2. Uma questão ampliada 4 vezes em uma aplicação Web utilizando CSS (a) e a mesma questão ampliada 4 vezes em uma aplicação Desktop utilizando o ampliador de telas presente no Windows 7 (b).

É importante observar que a escolha da tecnologia para desenvolvimento da ferramenta também deve considerar sua compatibilidade com softwares leitores de tela. No trabalho de Lazar et al [2007] o conflito entre o leitor de tela e a aplicação foi apontado como a segunda maior causa de frustração pelos usuários.

\section{Análise experimental}

Após identificarmos as técnicas apropriadas iniciamos a etapa de análise experimental, necessária para determinar se tais técnicas são capazes de entregar o conteúdo do exame corretamente. Para tanto, utilizamos questões da prova do ledor do exame de 2013. Este tipo de prova, fornecida pelo INEP, foi desenvolvida para atender especificamente deficientes visuais e contem a descrição textual de imagens, gráficos, equações e fórmulas. O software leitor de telas NVDA (NonVisual Desktop Access), em sua configuração padrão, foi utilizado para converter o texto das questões em fala, 
necessário para que pessoas com deficiência visual total tenham acesso ao conteúdo do exame.

Durante a análise identificamos que diversas questões, principalmente questões das áreas de exatas, não foram sintetizadas corretamente. Os problemas identificados podem ser divididos em dois grupos: problemas relacionados com a forma de apresentação do conteúdo da questão e problemas relacionados com a incapacidade do NVDA de sintetizar o conteúdo. Os problemas relacionados com a forma de apresentação da questão são problemas que podem ser solucionados facilmente. Um exemplo deste tipo de problema são os espaços em números encontrados em algumas questões. Em uma das questões, por exemplo, o texto "R\$ 1 200,00" é lido como "um real duzentos vírgula zero zero", ao invés de "mil e duzentos reais", devido ao espaço entre os números 1 e 200,00.

Problemas relacionados com a incapacidade do NVDA de sintetizar o conteúdo são mais difíceis de solucionar. A maior dificuldade está relacionada com a sinterização de símbolos, fórmulas e expressões matemáticas [Thurlow 2010], que é considerado por Sorge et al [2014] como um dos principais obstáculos para inclusão de deficientes visuais nas áreas de ciência, tecnologia, engenharia e matemática. Esta dificuldade também foi relatada por um participante que presta consultoria para empresas organizadoras de concursos, que a apontou como o maior obstáculo no uso do leitor de telas para conversão do conteúdo das provas em fala.

Poucos trabalhos apresentam propostas de ferramentas para converter automaticamente equações e fórmulas escritas com MathML em texto, para que possam ser lidas por softwares leitores de tela. Dentre estas ferramentas, destacamos o MathPlayer, desenvolvido pela Design Science. Suas limitações, no entanto, inviabilizam seu uso: é uma ferramenta proprietária, compatível somente com versões antigas do navegador Internet Explorer e não suporta o idioma português. Uma ferramenta de código aberto é descrita no trabalho de Sorge et al [2014], entretanto, o desenvolvimento de tal ferramenta encontra-se em estágio inicial e, assim como o MathPlayer, não há suporte para o idioma português.

A prova avaliada fornecia descrição textual de imagens e fórmulas químicas, mas não fornecia descrição textual das equações, fórmulas e símbolos presentes nas questões da área matemática e suas tecnologias, impossibilitando que o NVDA as sintetizasse corretamente. Portanto, fica evidente que todas as terminologias matemáticas e científicas utilizadas no exame deverão ser descritas textualmente, uma vez que os leitores de tela não proveem meios para sintetizar tais terminologias.

Com relação à ampliação dos elementos visuais utilizando CSS, o maior problema detectado foi a impossibilidade de ampliação do cursor. Embora o sistema operacional possua recursos de acessibilidade que permitam ampliar o cursor, sua ampliação é limitada, se comparado à ampliação realizada por meio de um software ampliador de tela. Uma alternativa encontrada para resolver esta limitação foi a criação de um mecanismo de ampliação do cursor na ferramenta de entrega do exame, ocultando o cursor padrão e emulando um cursor ampliado utilizando elementos HTML. 


\section{Desenvolvimento do protótipo}

Este trabalho contribuiu para a identificação de técnicas apropriadas para o desenvolvimento de uma ferramenta computacional de entrega do Enem capaz de atender as necessidades de pessoas com deficiência visual e apresentamos uma análise prévia que revelou desafios a serem explorados no uso de tais abordagens para entrega do conteúdo do exame. Esta identificação e análise foram fundamentais para o desenvolvimento de um protótipo funcional de uma ferramenta de entrega do exame para este público. Tal protótipo, juntamente com informações sobre o projeto, encontrase disponível em um sítio eletrônico específico ${ }^{1}$. Quando concluído o seu desenvolvimento, deficientes visuais de todo o Brasil poderão contribuir relatando, por meio de comentários e questionários, aspectos positivos e negativos da abordagem proposta, além de possíveis dificuldades encontradas durante seu uso.

\section{Próximas etapas}

Apesar de adotarmos recomendações consolidadas de usabilidade e acessibilidade no desenvolvimento do protótipo, não podemos garantir que os usuários conseguirão realizar o exame com sucesso sem uma avaliação com usuários reais. Portanto, a próxima etapa deste trabalho consiste na realização de uma avaliação de usabilidade, necessária para determinarmos a forma adequada de apresentação das informações e de interação, além de identificar possíveis problemas de acessibilidade e/ou usabilidade.

Para Nielsen "O teste com usuários reais é o método de usabilidade mais fundamental e em muitos casos insubstituível, uma vez que provê informações diretas sobre como as pessoas utilizam computadores e quais são os exatos problemas encontrados na interface testada" [Nielsen 1993]. Tais testes também são imprescindíveis para identificarmos conflitos entre a ferramenta e o software leitor de tela, uma vez que este problema foi apontado por Lazar et al [2007] como uma das maiores causas de frustração entre os usuários de leitores de tela. Esta avaliação de usabilidade, cujos procedimentos foram analisados e aprovados pelo CEP (Comitê de Ética em Pesquisa), será realizada com o apoio de alunos do ensino médio e profissionais deficientes visuais do Colégio Pedro II.

\section{Referências}

Barbosa, Waldelúcio. (2014) "Deficiente visual reclama das adaptações para realização de provas do Enem", http://www.meionorte.com/noticias/deficiente-visual-reclamadas-adaptacoes-para-realizacao-de-provas-do-enem-259855, Fevereiro.

Dolan, Robert et al. (2010) "Universal Design for Computer-Based Testing", http://images.pearsonassessments.com/images/tmrs/tmrs_rg/TMRS_RR_UDCBTGui delinesrevB.pdf, Fevereiro.

ETS. (2010) "How ETS Works to Improve Test Accessibility", https://www.ets.org/s/about/pdf/how_ets_works_improve_accessibility.pdf, Fevereiro.

Frankel, Lois. Kirsh, Barbara. (2014) "Development of the Computer-Voiced GRE®

\footnotetext{
${ }^{1}$ No endereço http://www.eneminclusivo.com.br.
} 
revised General Test for Examinees Who Are Blind or Have Low Vision", http://www.ets.org/s/research/pdf/gre_compendium.pdf, Fevereiro.

Hansen, Eric G. Forer, Douglas C. Lee, Moon J. (2004) "Toward Accessible ComputerBased Tests: Prototypes for Visual and Other Disabilities", https://www.ets.org/Media/Research/pdf/RR-04-25.pdf, Dezembro.

Hersh, Marion A. Johnson, Michael A. (2008) "Assistive Technology for Visually Impaired and Blind People”. United Kingdom, Springer-Verlag London Ltd.

INEP. (2014) "Enem 2014: Balanço de Inscrições", http://download.inep.gov.br/educacao_basica/enem/downloads/2014/balanco_inscric oes_enem_2014.pdf, Dezembro.

Ketterlin-Geller. Leanne R. (2005) "Knowing What All Students Know: Procedures for Developing Universal Design for Assessment". Em The Journal of Technology, Learning, and Assessment, 4(2). http://ejournals.bc.edu/ojs/index.php/jtla/article/ view/ 1649/ 1491, Janeiro.

Lazar, Jonathan et al. (2007) "What Frustrates Screen Reader Users on the Web: A Study of 100 Blind Users". Em International Journal of Human-Computer Interaction, 22(3), páginas 247-269. Lawrence Erlbaum Associates, Inc.

Ministério da Educação. (2002) "Exame Nacional do Ensino Médio: Documento básico", http://dominiopublico.mec.gov.br/download/texto/me000115.pdf, Dezembro.

Ministério da Educação. (2012) "O atendimento diferenciado no Enem", http://download.inep.gov.br/educacao_basica/enem/nota_tecnica/2012/atendimento_ diferenciado_enem_2012.pdf, Dezembro.

Nielsen, Jakob. (1993) “Usability Engineering”. San Diego, Academic Press.

Pernice, Kara. Nielsen, Jakob. (2001) "Usability Guidelines for Accessible Web Design”. http://www.nngroup.com/reports/usability-guidelines-accessible-webdesign/, Fevereiro.

Scheuermann, Friedrich. Pereira, Angela Guimarães. (2008) "Towards a research agenda on Computer-based Assessment", http://publications.jrc.ec.europa.eu/ repository/bitstream/111111111/907/1/reqno_jrc44526_report\%20final\%20version\% 5B2\%5D.pdf, Janeiro.

Sorge, Volker. Chen, Charles. Raman , T.V. Tseng, David. (2014) "Towards Making Mathematics a First Class Citizen in General Screen Readers". Em 14th Web for All Conference.

Thurlow, Martha et al. (2010) "Computer-based Testing: Practices and Considerations", Synthesis Report 78, University of Minnesota. http://files.eric.ed.gov/fulltext/ ED512613.pdf, Fevereiro.

Vieira, Leonardo. (2014) "Tenho medo da qualificação de quem lerá a prova do Enem para mim', diz deficiente visual", http:/oglobo.globo.com/sociedade/educacao/eneme-vestibular/tenho-medo-da-qualificacao-de-quem-lera-prova-do-enem-para-mimdiz-deficiente-visual-14439326, Janeiro. 